\title{
Mortality Modelling and Forecasting using Cross-Validation Techniques
}

\author{
Wouter van Wel*
}

\begin{abstract}
This paper investigates how accurately several predictive models perform in the field of mortallity modelling and forecasting, using cross-validation techniques. The main models used in this paper are the well-known Lee-Carter model and the Heligman-Pollard model. Furthermore, some extensions will be investigated: a distinction between the male and female population, extension to a few other countries, the effects of increasing the size of the "training set" and forecasting without re-estimating $k_{t}$ for the Lee-Carter model and a weighted estimation for the Heligman-Pollard model.
\end{abstract}

Keywords: Lee-Carter, Heligman-Pollard, mortality modelling, mortality forecasting, crossvalidation

\section{Introduction}

In the last century, human mortality rates have steadily declined, leading to longer life expectancies. Although, there are differences across gender, age and countries. Mortality trends have been affected by several events, such as the Spanish Flu and World War II, but also by changes in human behaviour, like smoking and alcohol consumption. Such things present risks for insurers, who might have not taken this into account. This is what makes mortality modelling, and in particular mortality forecasting, so important these days.

\footnotetext{
Wouter van Wel received a bachelor degree in Econometrics and Operations Research at Maastricht University in 2014. Currently he is taking a master in Econometrics and Operations Research at Maastricht University (specialisation: Econometrics).

Contact: w.vanwel@student.maastrichtuniversity.nl
} 


\section{Mortality Modelling and Forecasting using Cross-Validation Techniques}

Since the publication of the Gompertz law of mortality in 1825, several methods have been proposed for the modelling of mortality rates. However, this paper will only focus on the wellknown Lee-Carter model and the Heligman-Pollard model. The main objective is to investigate how accurately these two models will perform in practice, using Dutch data ${ }^{1}$. This will be done using cross-validation techniques. The first step is to divide the complete data set into two subsets: the first is used for analysis (modelling and forecasting), the latter for comparison [6].

Additional to the main analysis, a few extensions will be applied: a distinction between the male and female population, extension to Danish and Norwegian population, the effects of increasing the size of the "training set" and forecasting without re-estimating $k_{t}$ for the Lee-Carter model; and a weighted estimation for the Heligman-Pollard model. All these results will be compared with the original results for both models with Dutch data.

The paper is structured as follows. Chapter 2 provides an extensive description of the Heligman-Pollard model and the Lee-Carter model. Chapter 3 discusses the main analysis of these two models and compares their results. Chapter 4 adresses all extensions described before and discusses their results. Finally, Chapter 5 draws conclusions.

\section{Methods}

Nowadays, many methods have been proposed to model and forecast mortality rates, ranging from an extrapolative approach to GLM methods and the Lee-Carter method; for an extensive review, see Booth and Tickle [2]. The Lee-Carter is probably the best known method for mortality forecasting these days. Among the many parameterisation functions which have been proposed, the Heligman-Pollard model is well known.

\subsection{Heligman-Pollard Model}

The Heligman-Pollard model [3] is an eight-parameter parameterisation function. Parameterisation functions (laws of mortality) are one-factor models which try to depict the age pattern of mortality. However, they are of limited use in mortality forecasting due to unstable parameter time series and interdependencies [2]. The Heligman-Pollard model is defined as:

$$
\frac{q_{x}}{p_{x}}=A^{(x+B)^{C}}+D \exp \left[-E(\ln x-\ln F)^{2}\right]+G H^{x}
$$

where $A$ to $H$ are the eight parameters, and $p_{x}=1-q_{x}$.

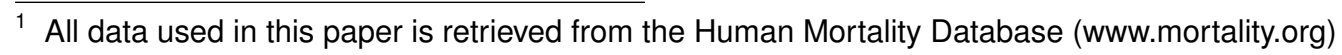




\section{Mortality Modelling and Forecasting using Cross-Validation Techniques}

The first component, a rapidly declining exponential, reflects the fall in mortality during the early childhood years. A measures the level of mortality, $C$ measures the rate of decline in childhood mortality and $B$ is an age displacement. The second component, a function similar to the lognormal, reflects accident mortality for male and accident plus maternal mortality for the female population. The "accident hump" can be found in all populations, usually between the ages of 10 and 40. $F$ indicates location, $E$ spread and $D$ the severity. The last component, also known as the Gompertz exponential, reflects the rise in mortality at adult ages, or senescent mortality. $G$ represents the base level of senescent mortality and $H$ reflects the rate of increase of that mortality. Figure 1 provides a nice illustration of all three components.

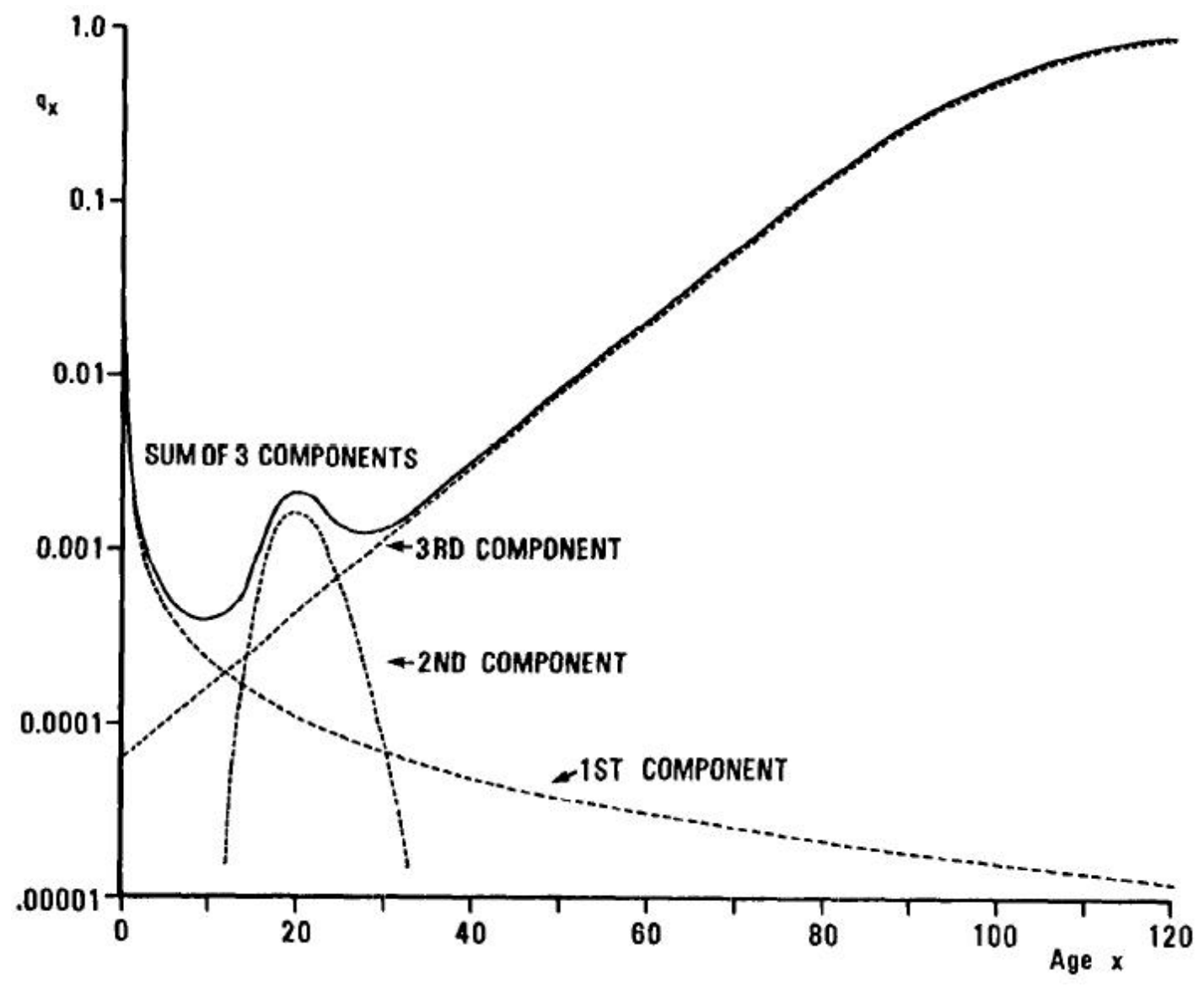

Figure 1 - The mortality curve and its three components. Australian national male mortality, 1970-72 [3].

Mortality forecasting using the Heligman-Pollard model is ideally done by multivariate time series methods. In this paper however, univariate time series methods are used. The time series for each of the eight parameters are obtained by repeatedly minimizing the following loss 


\section{Mortality Modelling and Forecasting using Cross-Validation Techniques}

function:

$$
\sum_{x=0}^{110}\left(\frac{q_{x}}{\dot{q}_{x}}-1\right)^{2}
$$

where $q_{x}$ is the fitted value at age $x$ and $\dot{q}_{x}$ is the observed mortality rate. The best ARIMA model is then fit to each parameter by looking at the Akaike Information Criterion (AIC) and the Bayesian Information Criterion (BIC). After forecasting each of the parameters separately, all forecasted parameters are plugged back into the Heligman-Pollard model to obtain the forecasted mortality rates.

\subsection{Lee-Carter Model}

The Lee-Carter model is a two-factor model specified by Lee \& Carter [5]. In contrast to parameterisation functions, which try to represent the age pattern of mortality, the Lee-Carter method is a principal components approach, whereby the age pattern is estimated from the data. The Lee-Carter model is specified as:

$$
\ln m_{x, t}=a_{x}+b_{x} k_{t}+\varepsilon_{x, t}
$$

where $m_{x, t}$ is the central mortality rate at age $x$ in year $t ; a_{x}$ is the average log-mortality at age $x ; b_{x}$ measures the response at age $x$ to change in $k_{t} ; k_{t}$ represents the overall level of mortality in year $t$; and $\varepsilon_{x, t}$ is the residual.

Estimation of the parameters is done as follows: $a_{x}$ is estimated by averaging log-mortality rates over time and $b_{x}$ and $k_{t}$ via singular value decomposition (SVD), a method for approximating a matrix as the product of two vectors. In order to get a unique solution, two constraints are necessary: the $b_{x}$ should sum to 1 and the $k_{t}$ to 0 . Without these constraints, the model in (2.3) is not identifiable. For example, the model with $\left(a_{x}, b_{x}, k_{t}\right)$ would be the same as the model with $\left(a_{x}, b_{x} / c, c k_{t}\right)$, for any constant $c$. Hereafter, the estimated $k_{t}$ is adjusted in such a way that the observed total deaths equal the total deaths "'predicted" by the model in each year. Doing so compensates the effect of taking log-rates, because ages at which deaths are high receive greater weights [1]. The adjusted estimated $k_{t}$ is modelled as a simple random walk with drift:

$$
k_{t}=k_{t-1}+d+e_{t}
$$

where $d$ is the drift parameter and $e_{t}$ is an error term. Forecasted mortality rates are then obtained by using the estimated $a_{x}$ and $b_{x}$ and forecast $k_{t}$. 


\section{Mortality Modelling and Forecasting using Cross-Validation Techniques}

\section{Main Analysis}

This chapter will discuss how the mortality rates are modelled and forecasted using the HeligmanPollard model and the Lee-Carter model. Furthermore, the results of both methods will be analysed and compared. In the main analysis, only Dutch data (total population) will be considered. The complete data set (1850-2009) is used, the period 1850-1979 is used as "training set" and the period 1980-2009 as "testing set". Figure 2 gives a graphical overview of the mortality curves for the Dutch population over the entire period from 1850 until 2009.

\section{Netherlands: total death rates (1850-2009)}

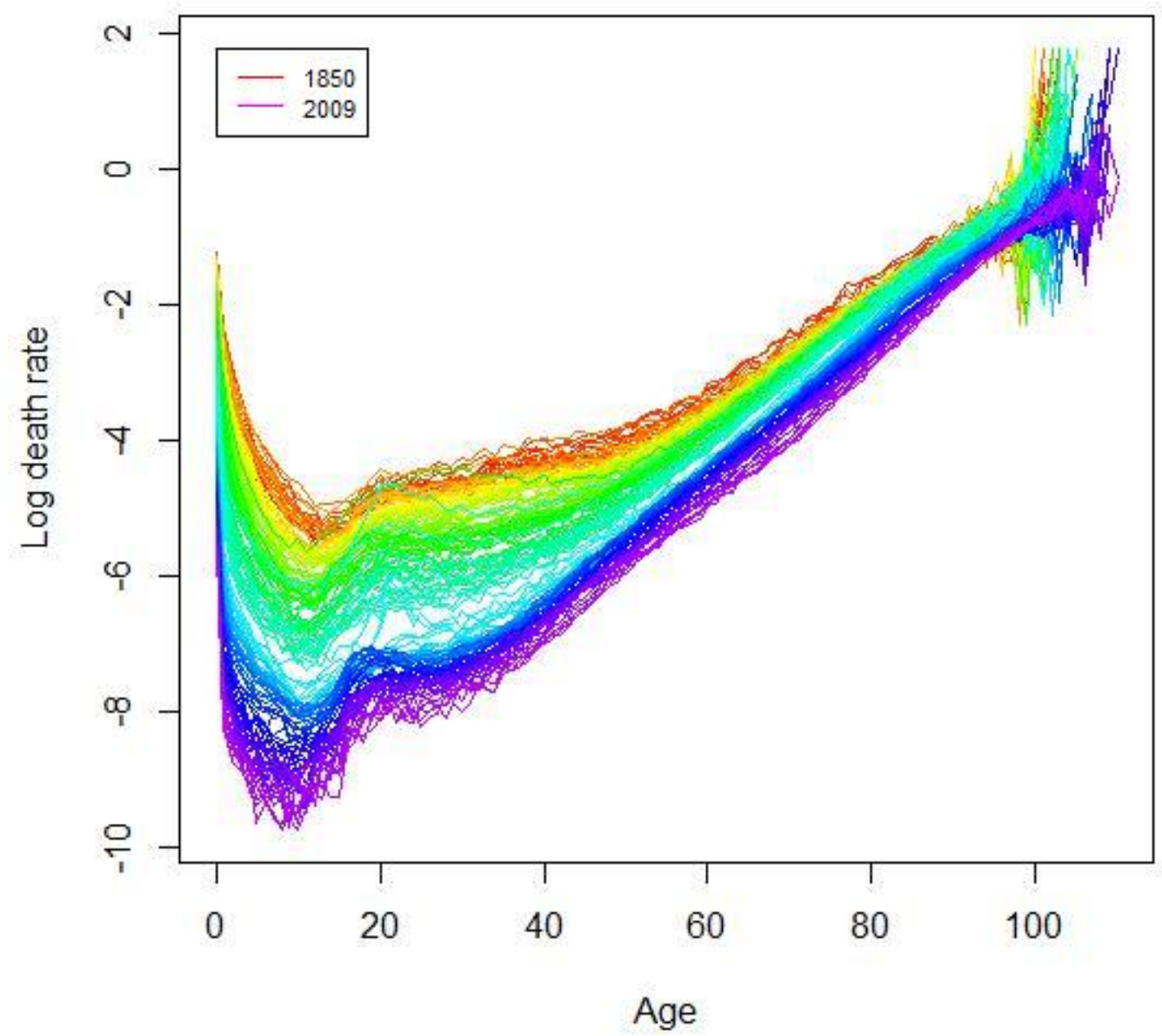

Figure 2 - The mortality curves for Dutch total population, period 1850-2009. 


\section{Mortality Modelling and Forecasting using Cross-Validation Techniques}

\subsection{Heligman-Pollard Model}

The complete estimation of the parameters for the Heligman-Pollard model has been done in Microsoft Excel, using its Solver function. Figure 4 shows time series graphs of all eight parameters. In some of the graphs, one will notice outliers in the years 1918-1919 and 1945. These are due to, respectively, the Spanish Flu and World War II. In order to receive a better forecast, these outliers have been removed.

Modelling and forecasting of the eight parameters has been done using Eviews. Univariate time series methods have been used to model all parameters according to ARIMA models. Table 1 provides a small overview of these ARIMA models.

\begin{tabular}{lllllllll}
\hline & $\log (\mathbf{A})$ & $\log (\mathbf{B})$ & $\mathbf{C}$ & $\log (\mathbf{D})$ & $\log (\mathrm{E})$ & $\mathbf{F}$ & $\log (\mathbf{G})$ & $\mathbf{H}$ \\
\hline ARIMA & $(2,1,2)$ & $(0,1,1)$ & $(3,1,4)$ & $(4,1,4)$ & $(4,1,4)$ & $(2,1,1)$ & $(2,1,1)$ & $(6,1,0)$ \\
AIC & -0.9301 & 0.4326 & -5.7585 & -0.8471 & -0.4825 & 2.5657 & -1.0587 & -9.3076 \\
BIC & -0.8164 & 0.4776 & -5.5756 & -0.6403 & -0.2757 & 2.6567 & -0.9677 & -9.1445 \\
\hline
\end{tabular}

Table 1 - ARIMA models for the parameters of the Heligman-Pollard model.

Using these ARIMA models, each parameter is forecasted. The forecasted mortality rates are then obtained by plugging these forecasted parameters back into the model. Time series graphs of all eight parameters with their forecast are illustrated in figure 3.4.

\subsection{Lee-Carter Model}

Forecasting mortality rates via the Lee-Carter model has been done using the demography package [4], available for R. Estimates for $a_{x}$ and $b_{x}$ and a time series graph for the adjusted $k_{t}$, with $95 \%$ confidence interval, are shown in Figure 3.
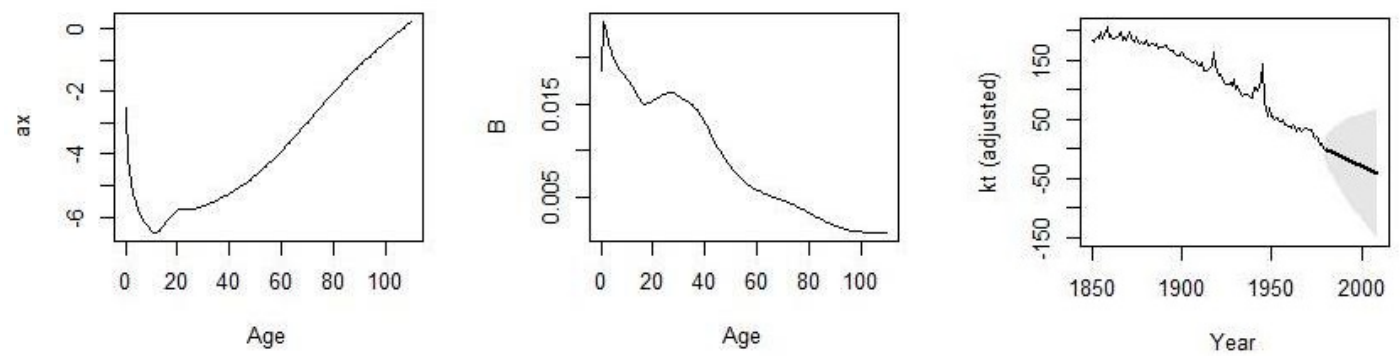

Figure 3 - Estimates for $a_{x}, b_{x}$ and the adjusted $k_{t}$ (Lee-Carter). 


\section{Mortality Modelling and Forecasting using Cross-Validation Techniques}

The left graph in Figure 3 shows the average over time log-mortality, whereas the middle graph shows how a certain age group responds to a change in the adjusted $k_{t}$, which is illustrated in the lower-right graph.

The graphs in Figure 6 and 7 give a small illustration of how the actual and forecasted mortality curve of the Dutch population in the first year (1980) and the last year (2009) of the forecasting period look like.

\subsection{Comparison}

The comparison of the Heligman-Pollard and Lee-Carter model for Dutch data will be based on the mean absolute percentage error (MAPE). Figure 8 and 9 show the MAPE of the log-mortality for both models. A big disadvantage of the Heligman-Pollard model is that big errors occur at ages 0 and 110+, which obviously can be seen from the graph in Figure 8 . The Lee-Carter model does not have this disadvantage, but seems to have higher errors at adolescent ages. Comparing their overall fit, the Heligman-Pollard model seems to fit better to the Dutch data, which is a striking result, since the Heligman-Pollard model is supposed to be worse in forecasting than the Lee-Carter model. 
Mortality Modelling and Forecasting using Cross-Validation Techniques

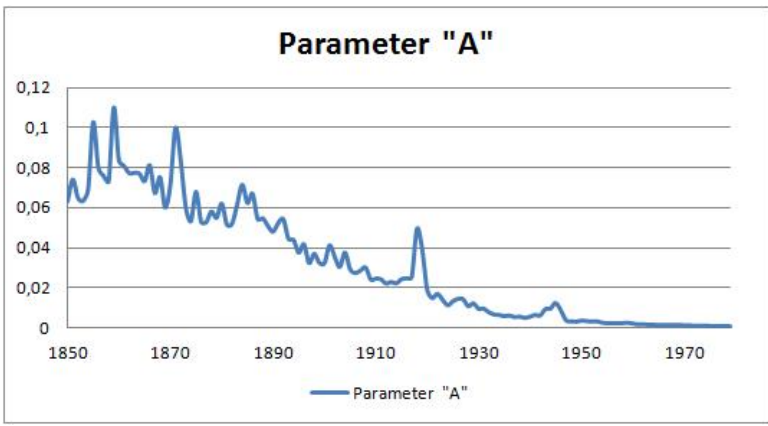

(a) Parameter $\mathrm{A}$

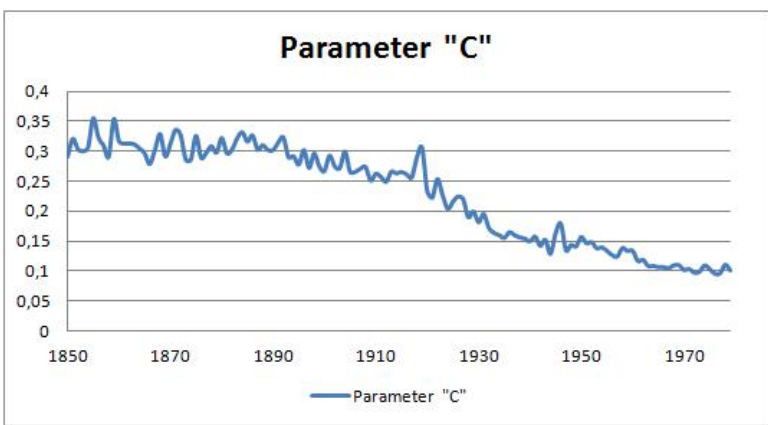

(c) Parameter C

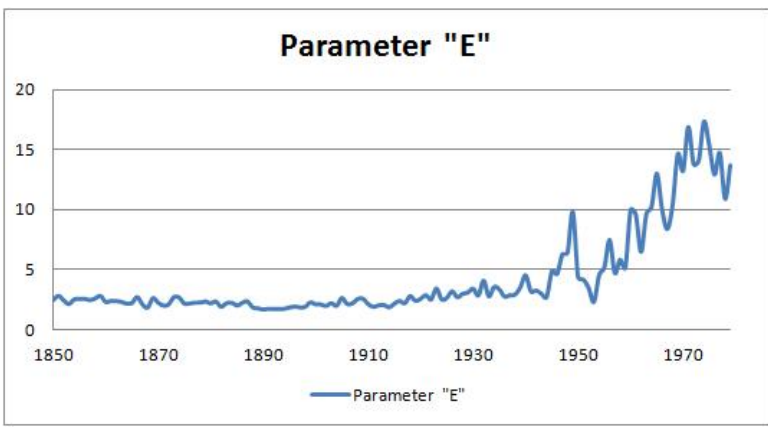

(e) Parameter E

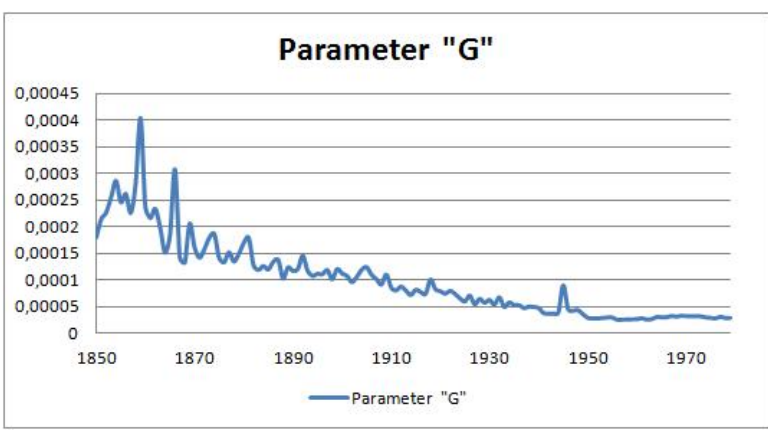

(g) Parameter G

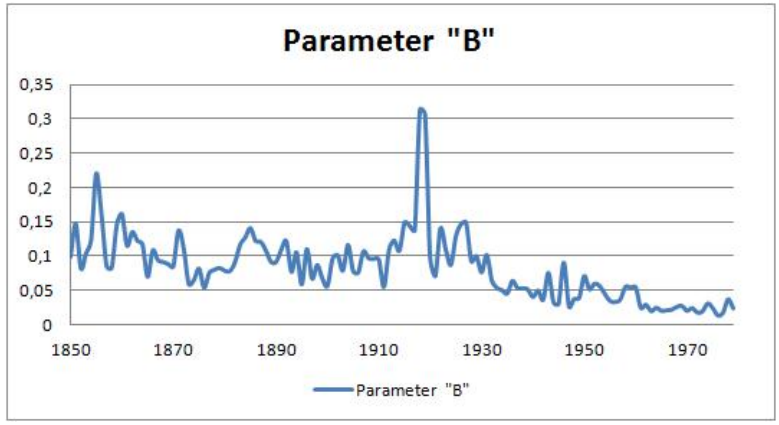

(b) Parameter B

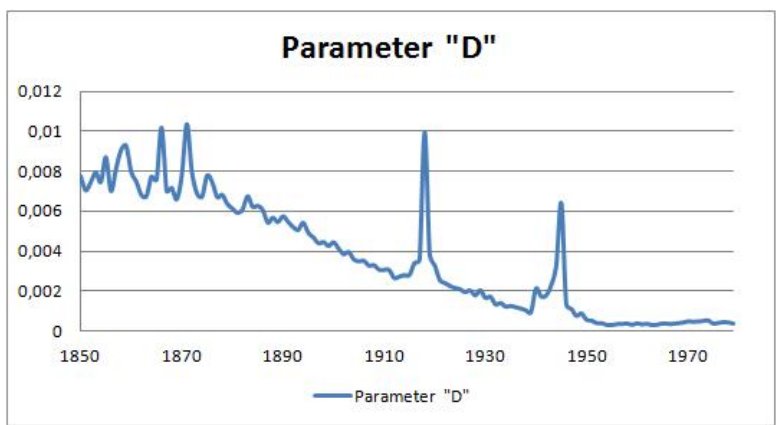

(d) Parameter D

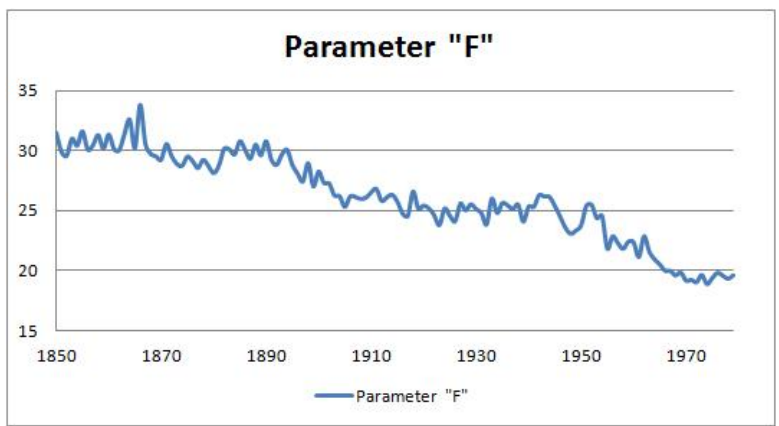

(f) Parameter F

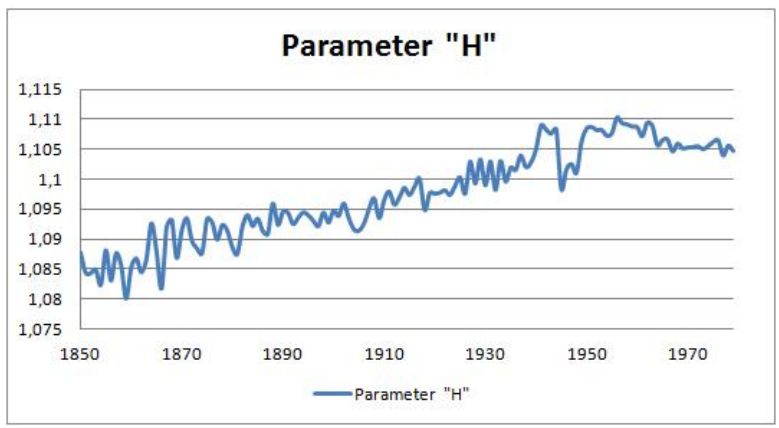

(h) Parameter $\mathrm{H}$

Figure 4 - Time series of all eight parameters (Heligman-Pollard).

Marble series (2015) 1: Quantitative methods in Business and Economics 
Mortality Modelling and Forecasting using Cross-Validation Techniques

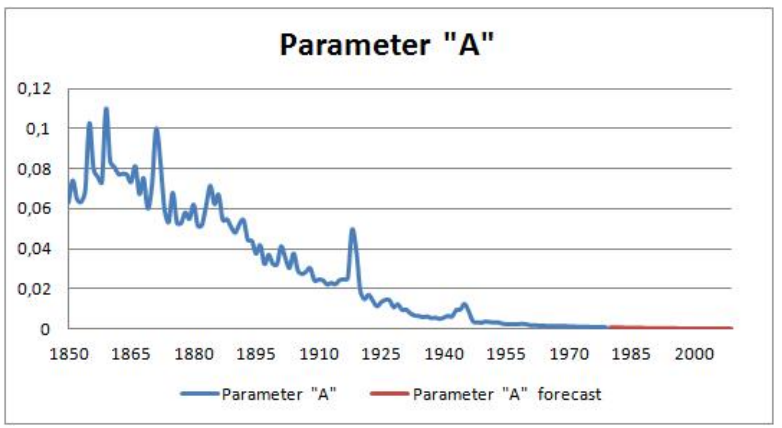

(a) Parameter $A$ with forecast

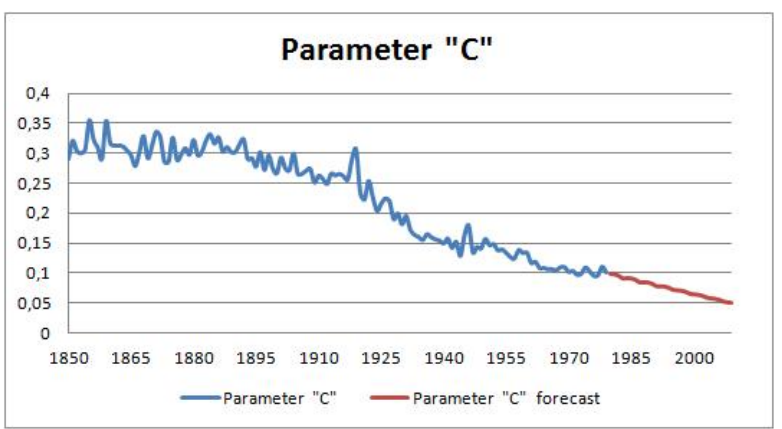

(c) Parameter $\mathrm{C}$ with forecast

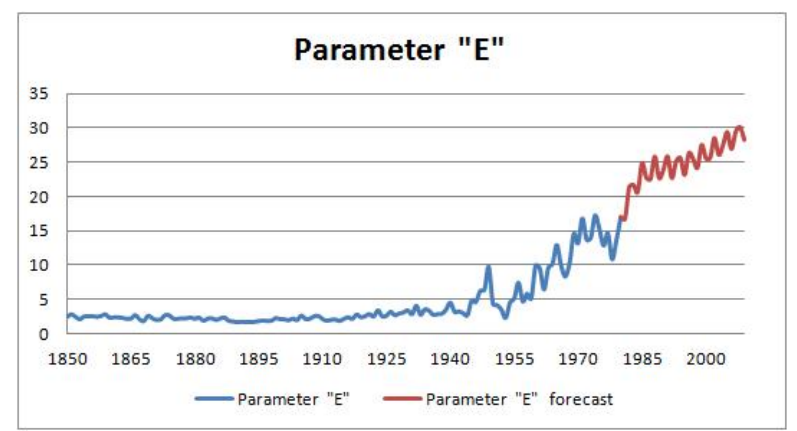

(e) Parameter E with forecast

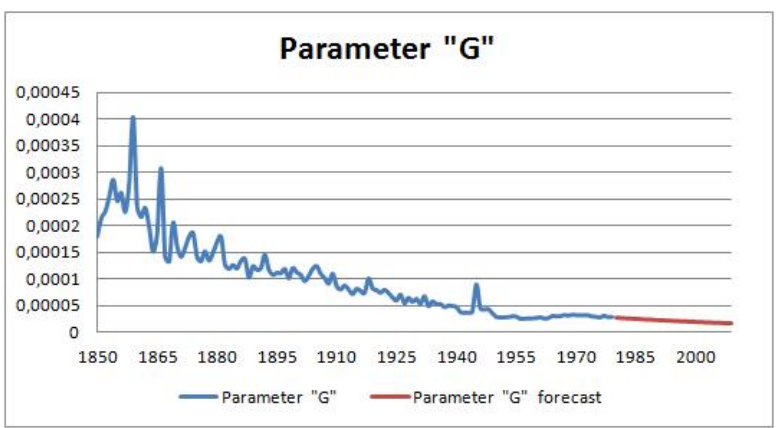

(g) Parameter $G$ with forecast

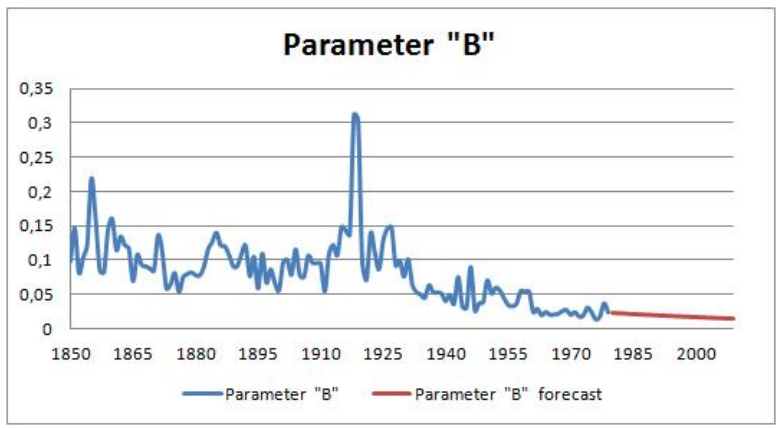

(b) Parameter B with forecast

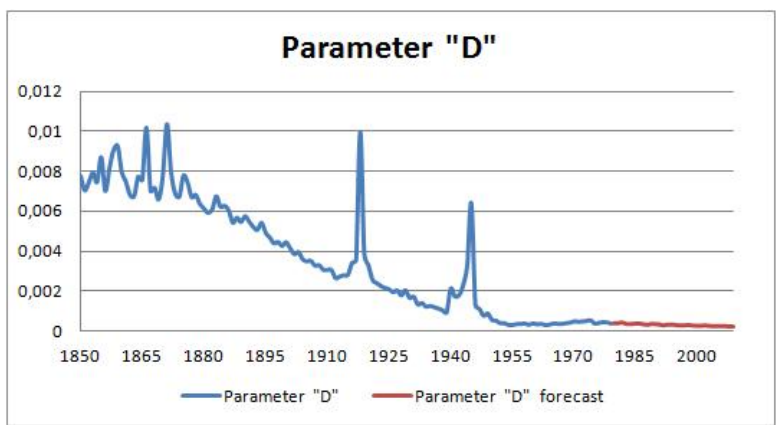

(d) Parameter D with forecast

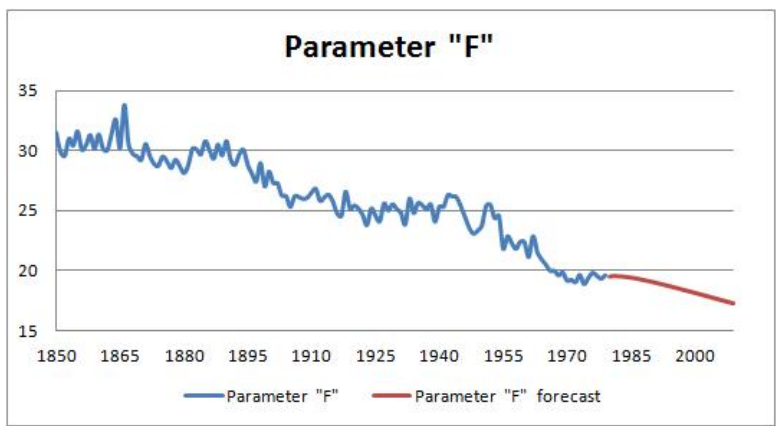

(f) Parameter F with forecast

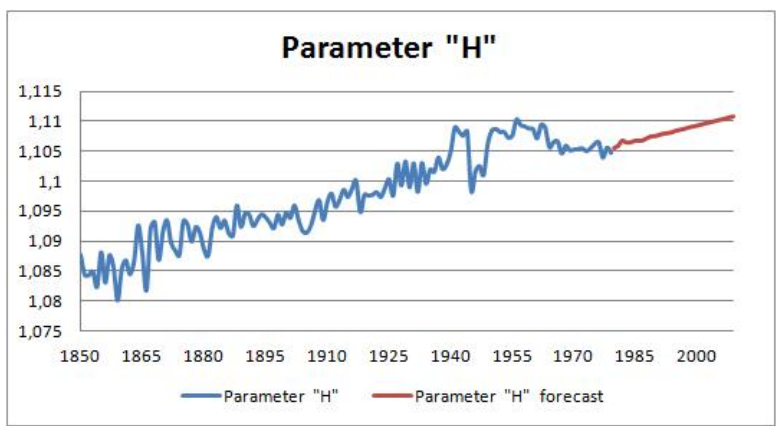

(h) Parameter $\mathrm{H}$ with forecast

Figure 5 - Time series with forecast of all eight parameters (Heligman-Pollard). 


\section{Mortality Modelling and Forecasting using Cross-Validation Techniques}
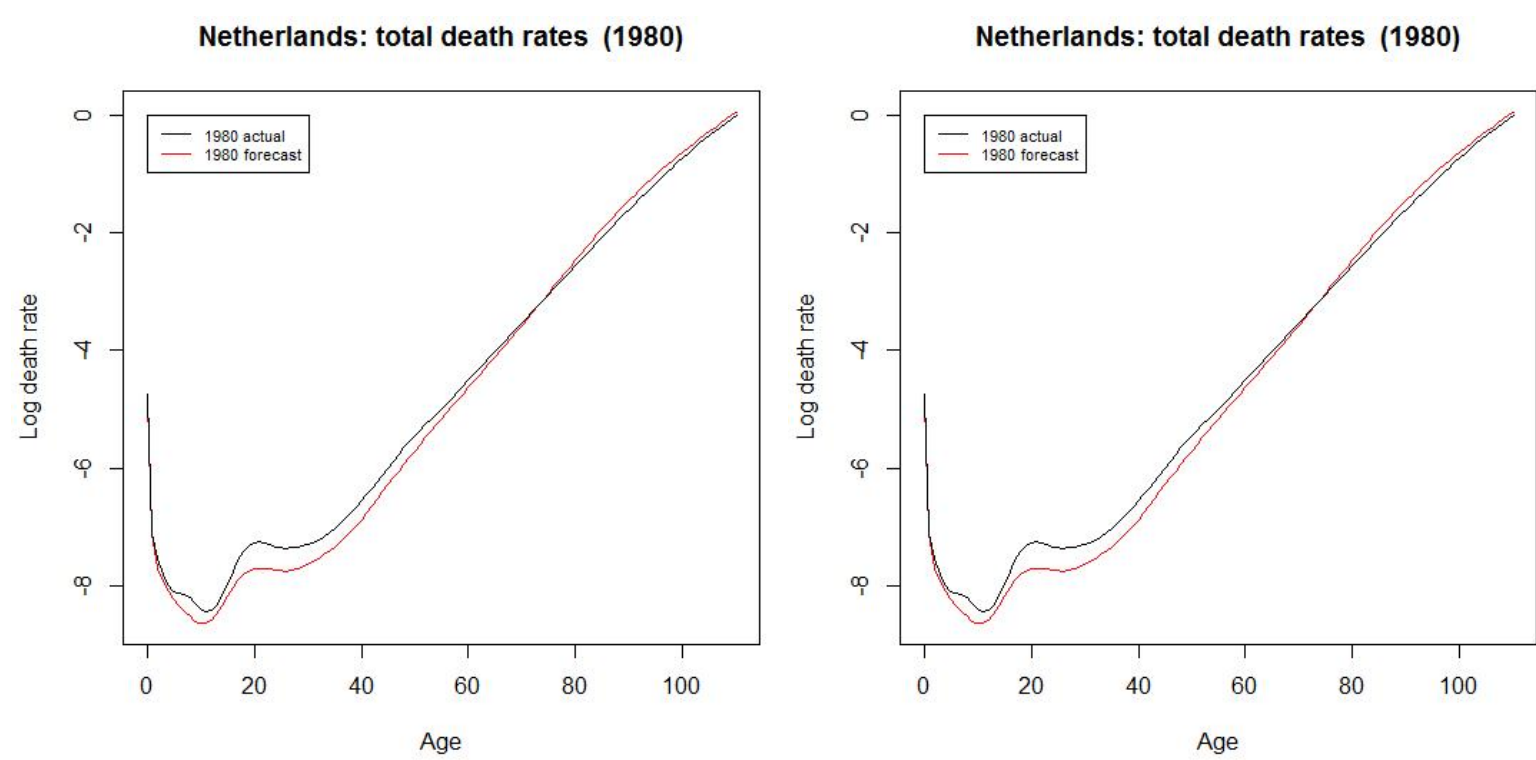

Figure 6 - Actual and forecasted mortality curve for the Netherlands in 1980 (Lee-Carter).

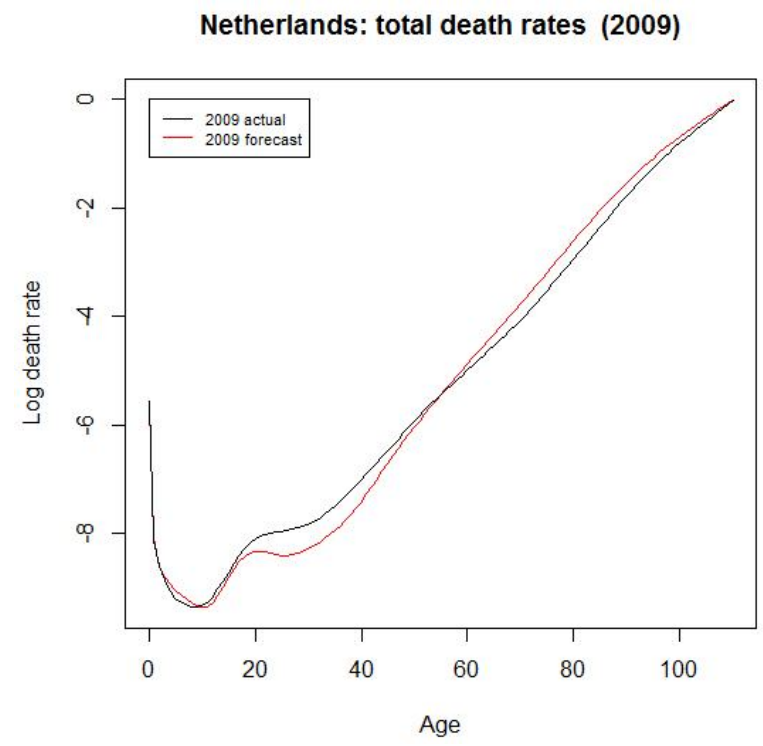

Figure 7 - Actual and forecasted mortality curve for the Netherlands in 2009 (Lee-Carter). 
Mortality Modelling and Forecasting using Cross-Validation Techniques

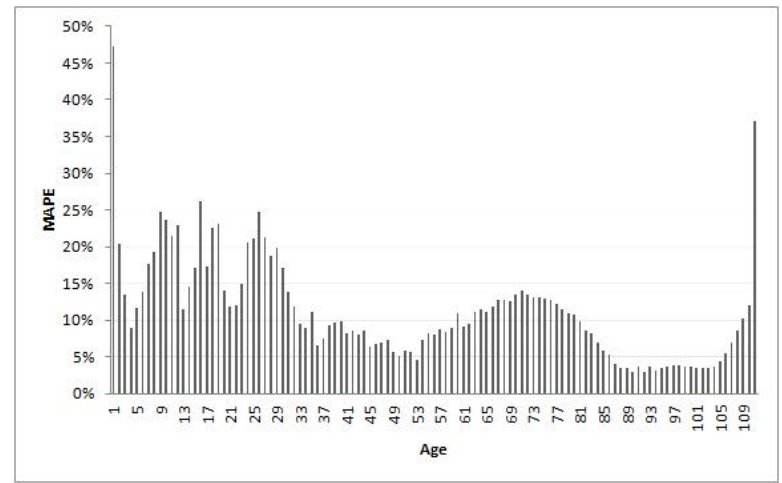

Figure 8 - MAPE Heligman-Pollard for Netherlands, total population (1980-2009).

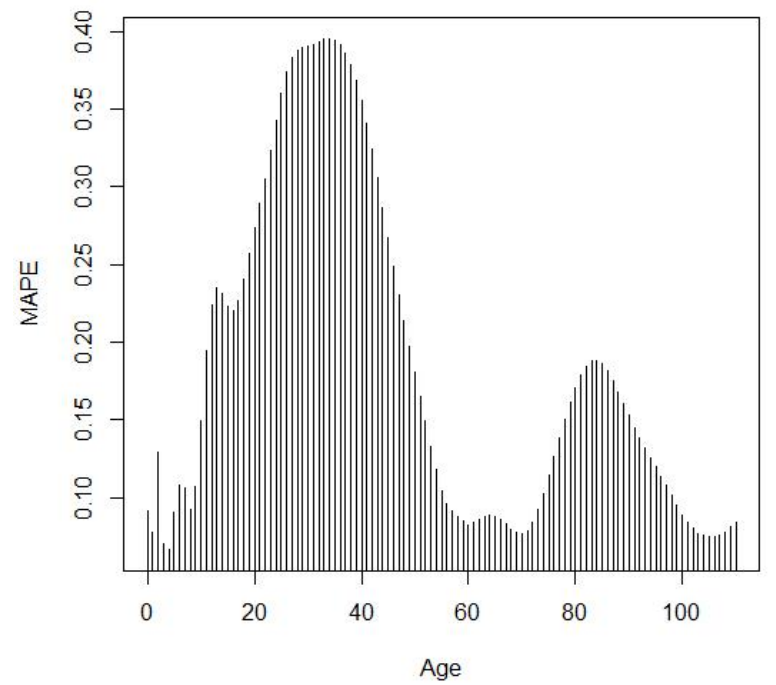

Figure 9 - MAPE Lee-Carter for Netherlands, total population (1980-2009). 


\section{Mortality Modelling and Forecasting using Cross-Validation Techniques}

\section{Extensions}

This chapter will elaborate on the extensions mentioned before. For the Heligman-Pollard model, a weighted estimation will be investigated. For the Lee-Carter model, a distinction between male and female population will be made for the Dutch data, Denmark and Norway will be used for forecasting, the effect of increasing the "training set" will be considered and forecasts will be made without re-estimating the $k_{t}$ series.

\subsection{Heligman-Pollard Extensions}

\subsubsection{Weighted Heligman-Pollard Estimation}

In Chapter 3, we noticed big errors at ages 0 and 110+ for the Heligman-Pollard model. In order to reduce these errors, a weighted estimation is used. The weights are defined by the number of deaths in a certain year for a certain age group $\left(d_{x, t}\right)$, as proposed by Wilmoth [7]. Since the variance of $\ln m_{x, t}$ is roughly $1 / d_{x, t}$, bigger weights should be given to observations with a higher value of $d_{x, t}$, since these observations have a lower variance. Table 2 provides an overview of the ARIMA models selected for each parameter in case of a weighted estimation.

\begin{tabular}{lllllllll}
\hline & $\log (\mathbf{A})$ & $\log (\mathbf{B})$ & $\log (\mathbf{C})$ & $\log (\mathbf{D})$ & $\log (\mathrm{E})$ & $\mathbf{F}$ & $\log (\mathbf{G})$ & $\mathbf{H}$ \\
\hline ARIMA & $(2,1,2)$ & $(4,1,3)$ & $(4,1,3)$ & $(4,1,4)$ & $(4,1,4)$ & $(3,1,4)$ & $(3,1,3)$ & $(0,1,1)$ \\
AIC & -0.8046 & 0.3330 & -2.2375 & -0.8606 & 0.5507 & 4.3226 & -0.7185 & -8.9368 \\
BIC & -0.6909 & 0.5169 & -2.0536 & -0.6538 & 0.7576 & 4.5055 & -0.5585 & -8.8918 \\
\hline
\end{tabular}

Table 2 - ARIMA models for the parameters of the weighted Heligman-Pollard model.

The results of using a weighted estimation are illustrated in Figure 10. The purpose of this adjustment seems not to be fully achieved; the big error at age 0 has been reduced significantly, but the error at age 110+ has even slightly increased, this being the results of a relatively low weight for age group 110+, as there are few deaths at this age. Furthermore, large errors at age 14 and 15 arise, almost reaching $50 \%$. Besides these two big errors, this adjustment seems to reduce the errors at ages $0-20$, but performs worse at ages 21-70. Considering ages 71-110+, the weighted estimation seems to slightly reduce the errors, except for ages 89-105 and $110+$. The overall fit seems to be slightly worse than before, leading to the conclusion that the Heligman-Pollard model without weights seems to fit better to the Dutch data. 


\section{Mortality Modelling and Forecasting using Cross-Validation Techniques}

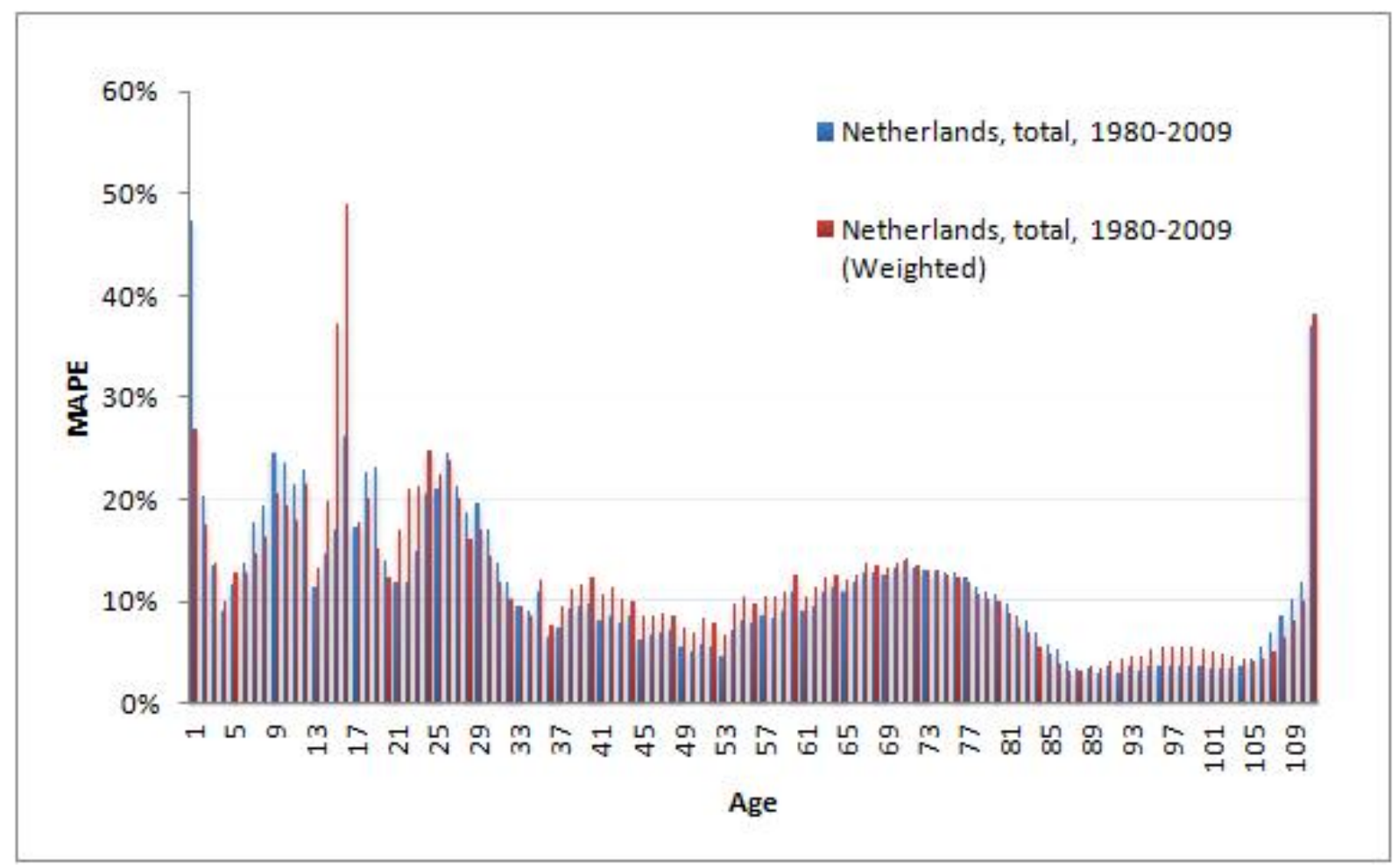

Figure 10 - Comparison MAPE Heligman-Pollard unweighted/weighted for Netherlands, total population (1980-2009).

\subsection{Lee-Carter Extensions}

\subsubsection{Distinction between male and female population}

The first extension which will be investigated is the distinction between male and female population for the Dutch data. The length of the "training" and "testing" set will remain the same. The time-averages of $a_{x}$ and $b_{x}$ and the time series of the adjusted $k_{t}$ for the Dutch male and female population are reported in respectively Figure 11 and 12.

The comparison for this extension is based on the mean percentage error (MPE). Figure 13 illustrates the MPE for the Dutch male and female population. The result is kind of strange. For the ages $0-20$, the MPE for the male population shows high positive errors up to approximately $120 \%$, then slowly fading to 0 . For ages $40-110+$, the male MPE seems to fluctuate around $10 \%$. However, for the female population, one observes high negative errors at ages 0-60, up to approximately $-60 \%$, then fading to 0 aswell. For the ages $70-110+$, the female MPE shows positive errors up to $20 \%$. 


\section{Mortality Modelling and Forecasting using Cross-Validation Techniques}
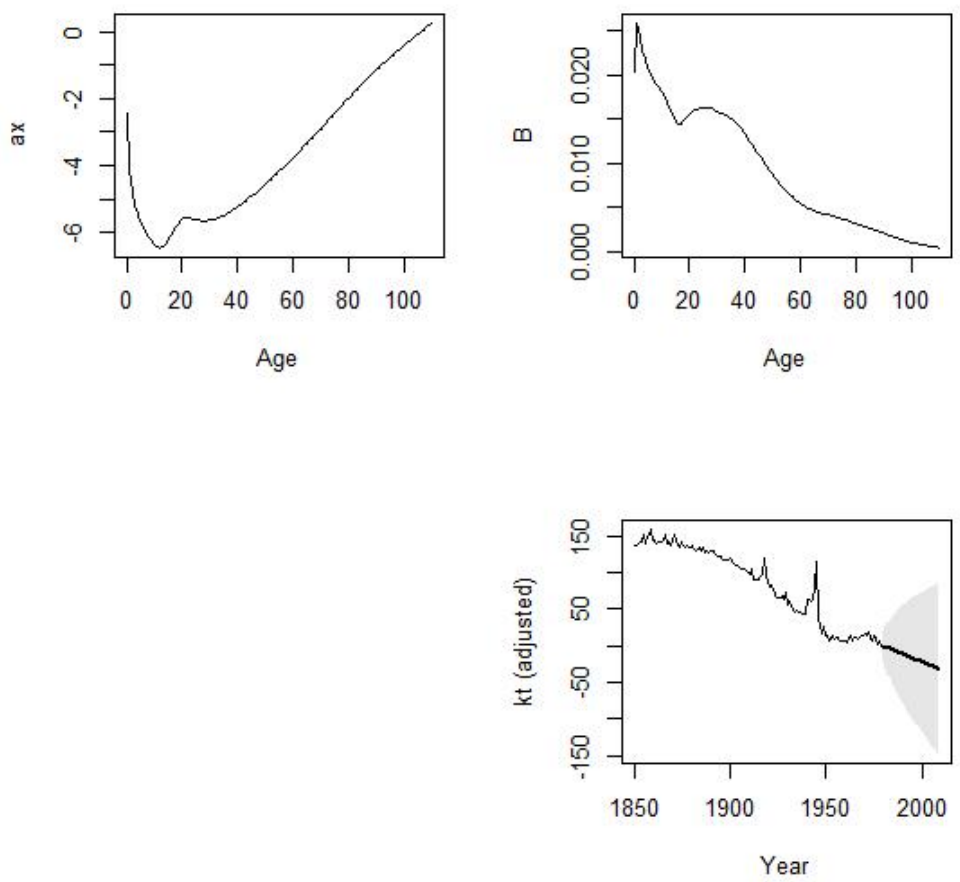

Figure 11 - Estimates for $a_{x}, b_{x}$ and the adjusted $k_{t}$ for the Dutch male population.

\subsubsection{Extension to Denmark and Norway}

The second extensions of the Lee-Carter model includes forecasting using Danish and Norwegian data. These two countries were picked for a certain reason, namely that they cover the same time-horizon as the Dutch data, whereas for all other countries, the data starts after 1850. In this way, and by having the same length of the "training" and "testing" set for all three countries, it makes sense to compare the results. The time-averages of $a_{x}$ and $b_{x}$ and the time series of the adjusted $k_{t}$ for the Danish and Norwegian population are reported in respectively Figure 14 and 15.

The comparison for this extension is based on the MAPE. Figure 16 shows the MAPE for the Netherlands, Denmark and Norway (total population). The big error hump at adolescent ages appears to be smaller for both Norway and Denmark, for the latter it has even decreased untill approximately $40 \%$. Considering ages $70-90$, both Norway and Denmark have bigger errors than the Netherlands. At ages 100-110+, the MAPE's for Denmark and the Netherlands are close to eachother, but Norway seems to produce a bit higher errors. We can conclude that the Norwegian data seems to overall fit slightly better than the Dutch data. Although, the Lee-Carter model seems to fit best to the Danish data, where the MAPE at adolescent ages is considerably lower. 


\section{Mortality Modelling and Forecasting using Cross-Validation Techniques}
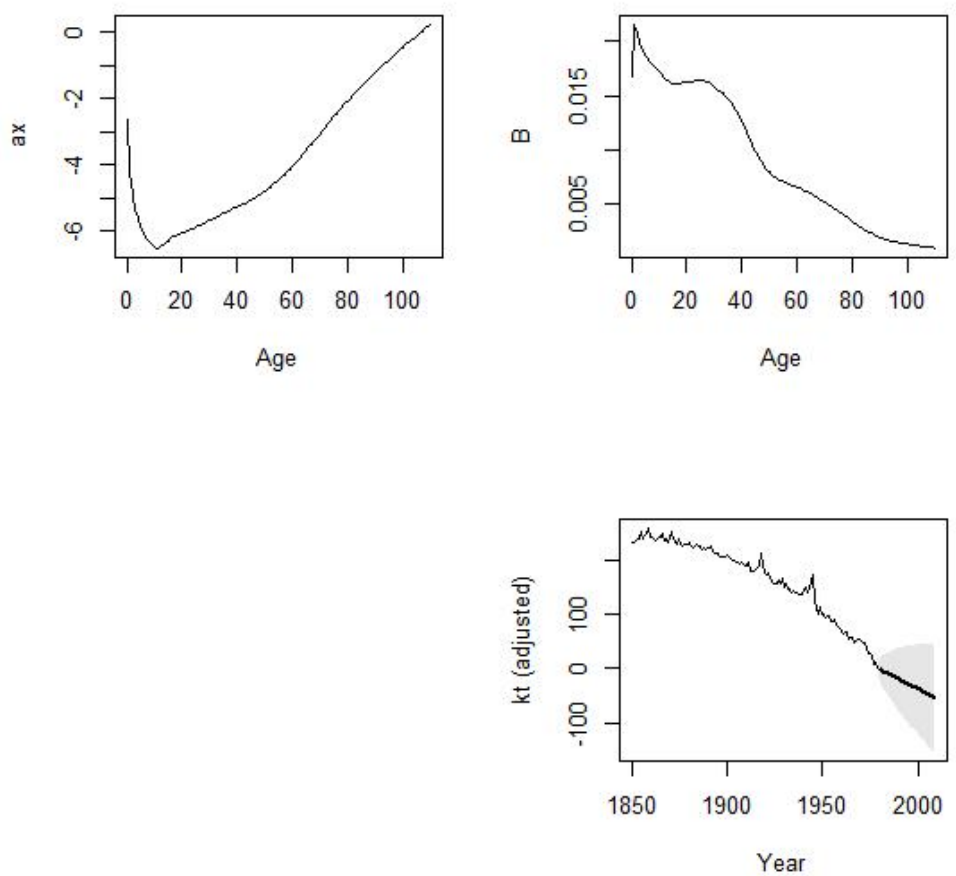

Figure 12 - Estimates for $a_{x}, b_{x}$ and the adjusted $k_{t}$ for the Dutch female population.

\subsubsection{Increasing the "training set"}

This extension of the Lee-Carter looks into the effect of increasing the "training set". The Dutch data will be used again and the "training set" will be increased twice, once by 10 years and once by 20 years. One would expect that increasing the "training set" would reduce the errors, as there is more information available for analysis (i.e. modelling and forecasting). The time-averages of $a_{x}$ and $b_{x}$ and the time series of the adjusted $k_{t}$ for both the 10 and 20 years increased "training sets" are reported in respectively Figure 17 and 18.

The comparison for this extension is again based on the MAPE and is illustrated in Figure 19. The red line shows the MAPE from the main analysis, as in Chapter 3 . The black and green line respresent, respectively, the 10 and 20 years increase of the "training set". This means that instead of forecasting over a period of 30 years (1980-2009), now only 20 (1990-2009) and 10 years (2000-2009) are being forecasted. Looking at the 10 years increase, there is a slight decrease of errors at ages 0-60 and a somewhat bigger decrease at ages 80-110+. Although, there is a small increase at ages 65-75. Considering the 20 years increase, the errors at adolescent ages seem to decrease even more and there's also a slight decrease at ages 80-110+ compared with the 10 years increased "training set". Although, at ages $60-80$ there is a significant increase in the MAPE. So, increasing the length of the "training set" seems give 


\section{Mortality Modelling and Forecasting using Cross-Validation Techniques}

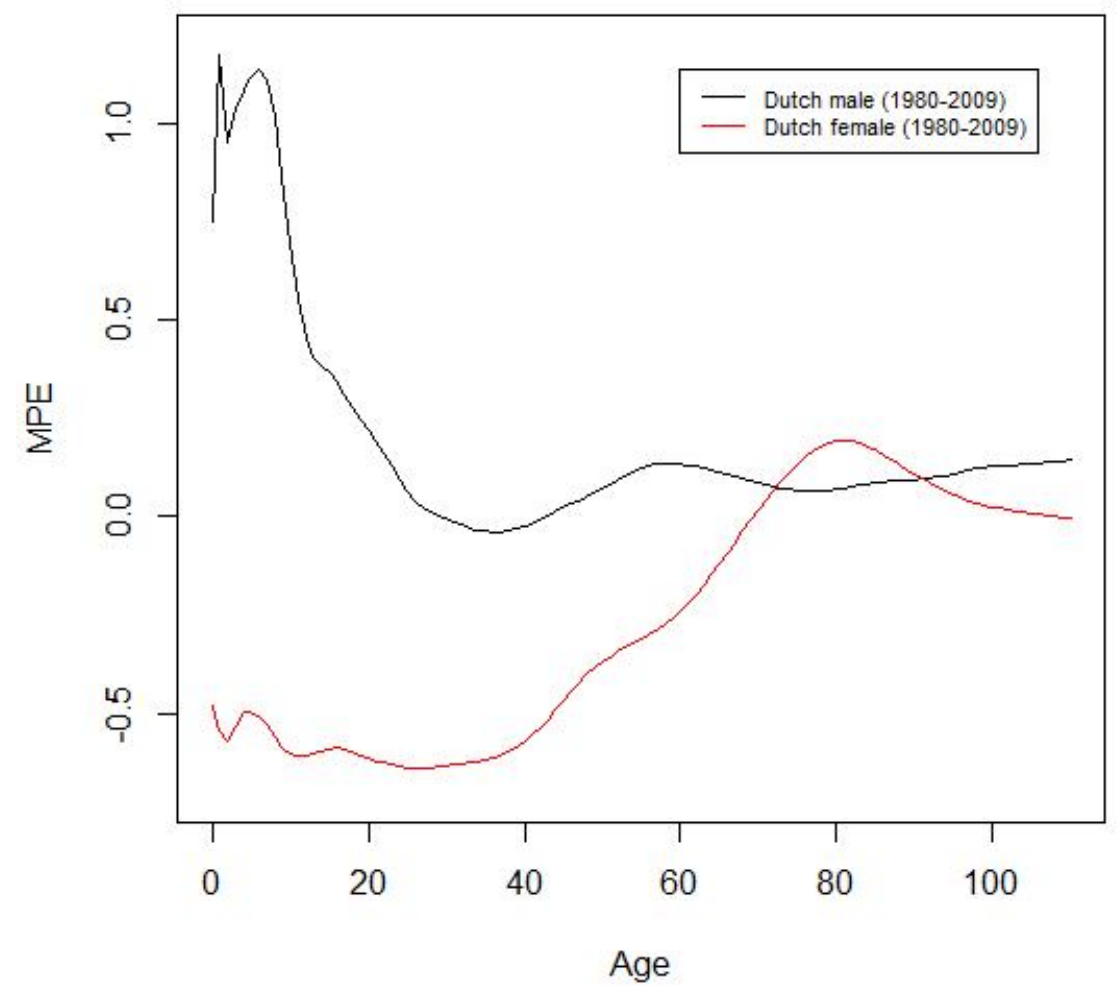

Figure 13 - Comparison MPE Lee-Carter for Netherlands, male and female population (1980-2009).

better forecasts using the Lee-Carter model, although for ages around 60-80 the MAPE seems to increase.

\subsubsection{Forecasting without adjusting the $k_{t}$ series}

Every Lee-Carter forecast in this paper used the adjustment for the $k_{t}$ series, as described in section 2.2. This section will investigate the effect of not using this adjustment for the Dutch data. One would expect that errors will rise for age groups with a relative high number of deaths, because their weights will now be lower. Hence, the observed number of deaths will now not be equal to the number of deaths predicted by the model. Figure 20 shows the time-averages of $a_{x}$ and $b_{x}$ and the time series $k_{t}$, without adjustment.

The comparison for the last extension is again based on the MAPE and is illustrated in Figure 21. The results show what we expected: a rise in the MAPE for age groups with a relative high number of deaths. There is a significant larger MAPE for ages 0-10 and also for ages $60-110+$ there is an increase. For the adolescent ages, we observe considerably lower errors, where the top only reaches $20 \%$ now. 
Mortality Modelling and Forecasting using Cross-Validation Techniques
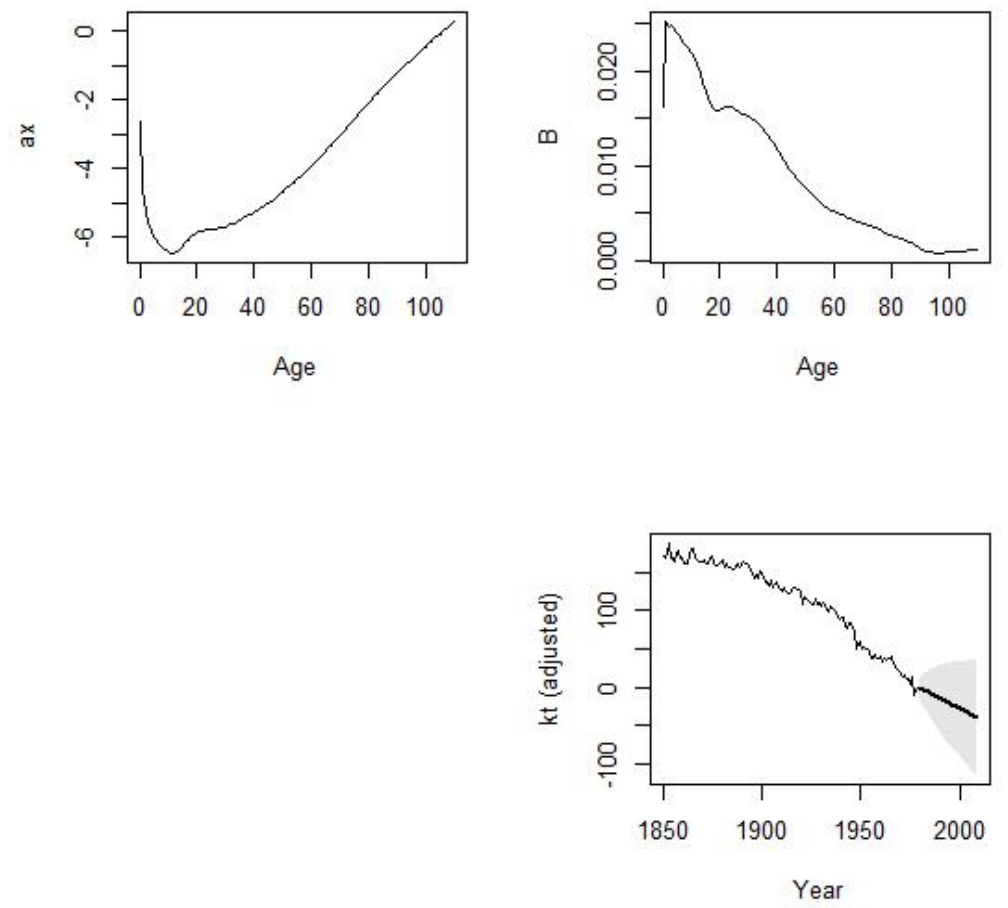

Figure 14 - Estimates for $a_{x}, b_{x}$ and the adjusted $k_{t}$ for Denmark, total population.
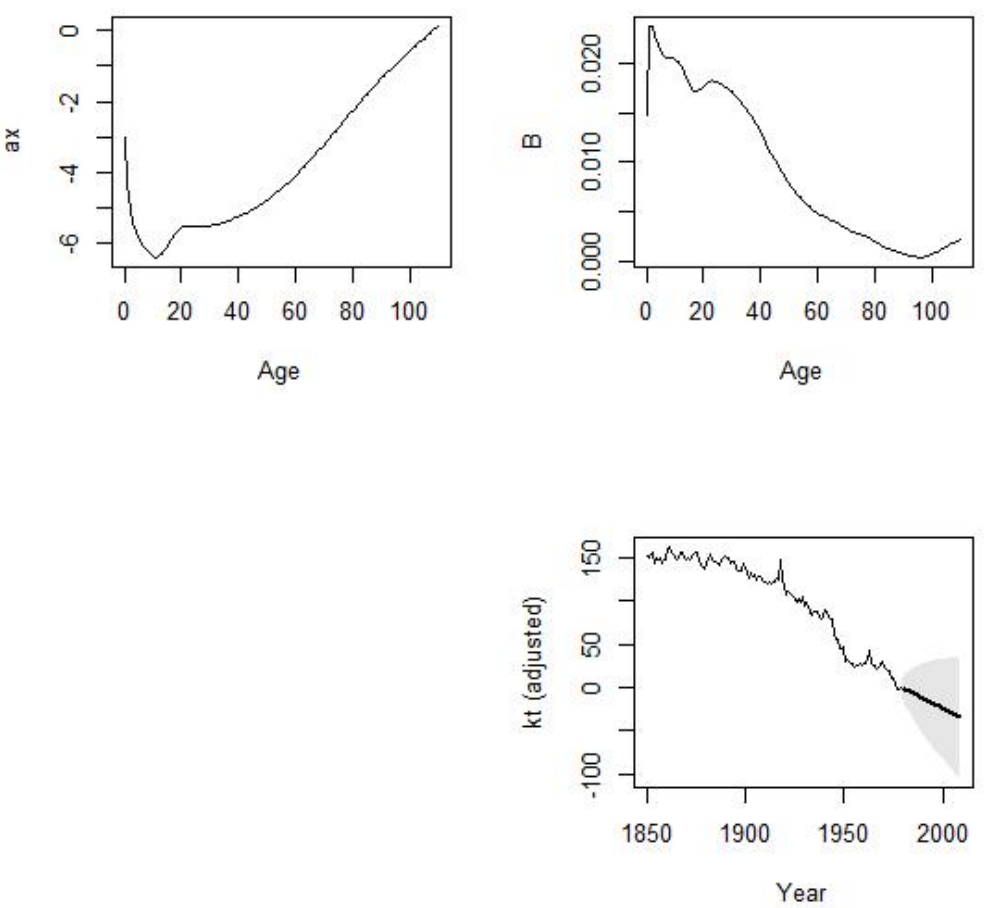

Figure 15 - Estimates for $a_{x}, b_{x}$ and the adjusted $k_{t}$ for Norway, total population. 
Mortality Modelling and Forecasting using Cross-Validation Techniques

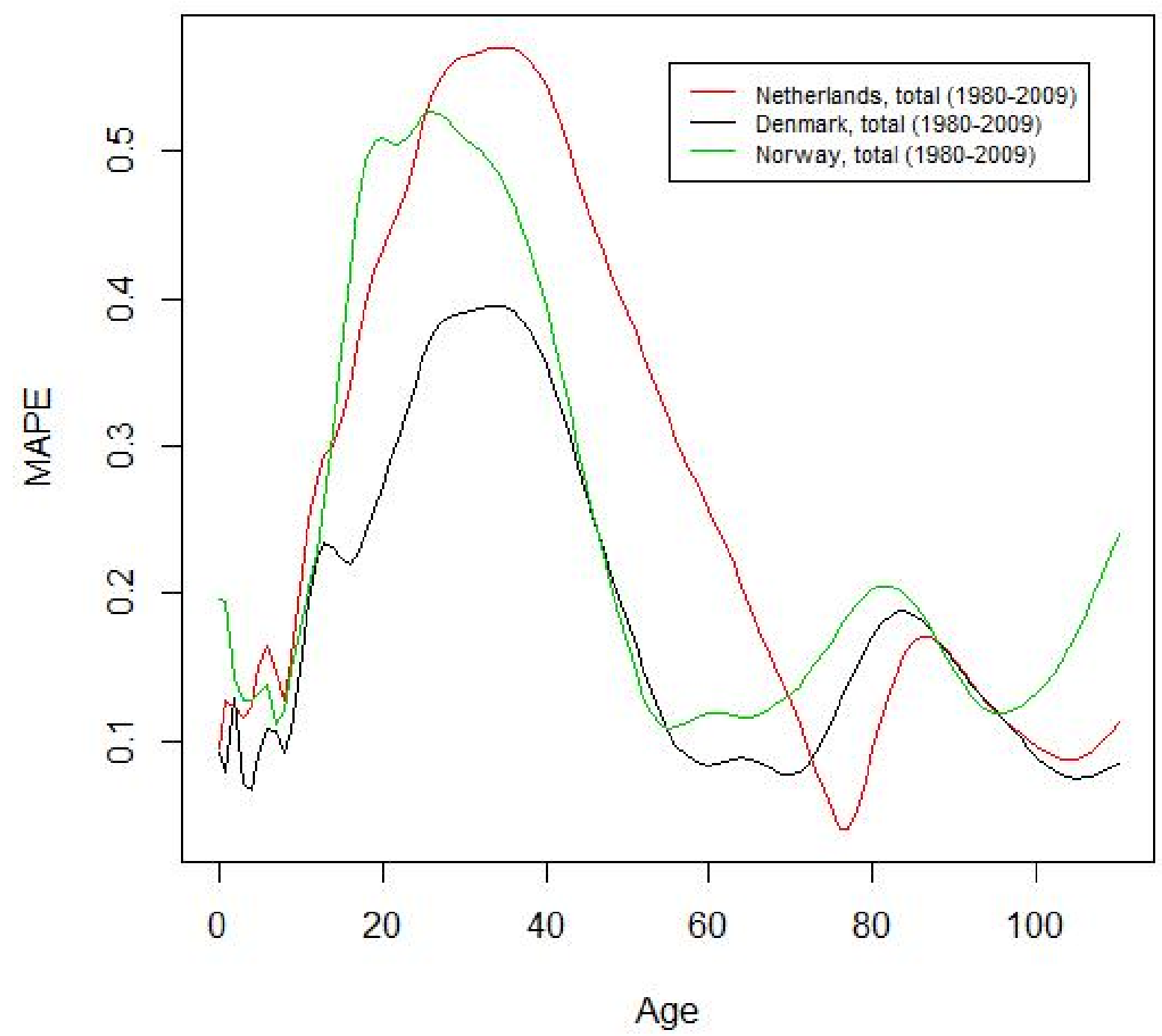

Figure 16 - Comparison MAPE Lee-Carter for the Netherlands, Denmark and Norway, total population (1980-2009). 
Mortality Modelling and Forecasting using Cross-Validation Techniques
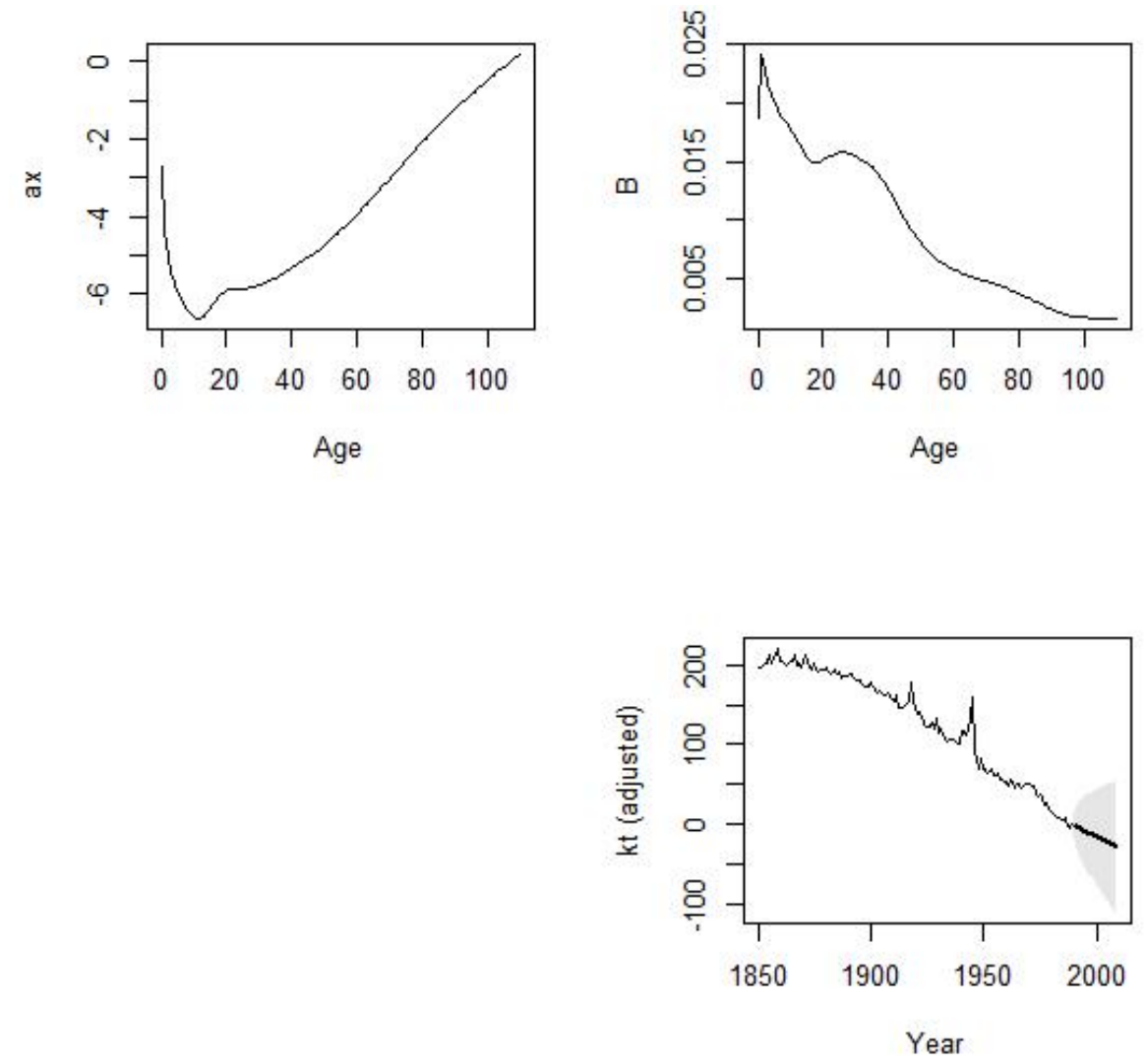

Figure 17 - Estimates for $a_{x}, b_{x}$ and the adjusted $k_{t}$ for the Netherlands, total population, "training set" 1850-1989. 
Mortality Modelling and Forecasting using Cross-Validation Techniques
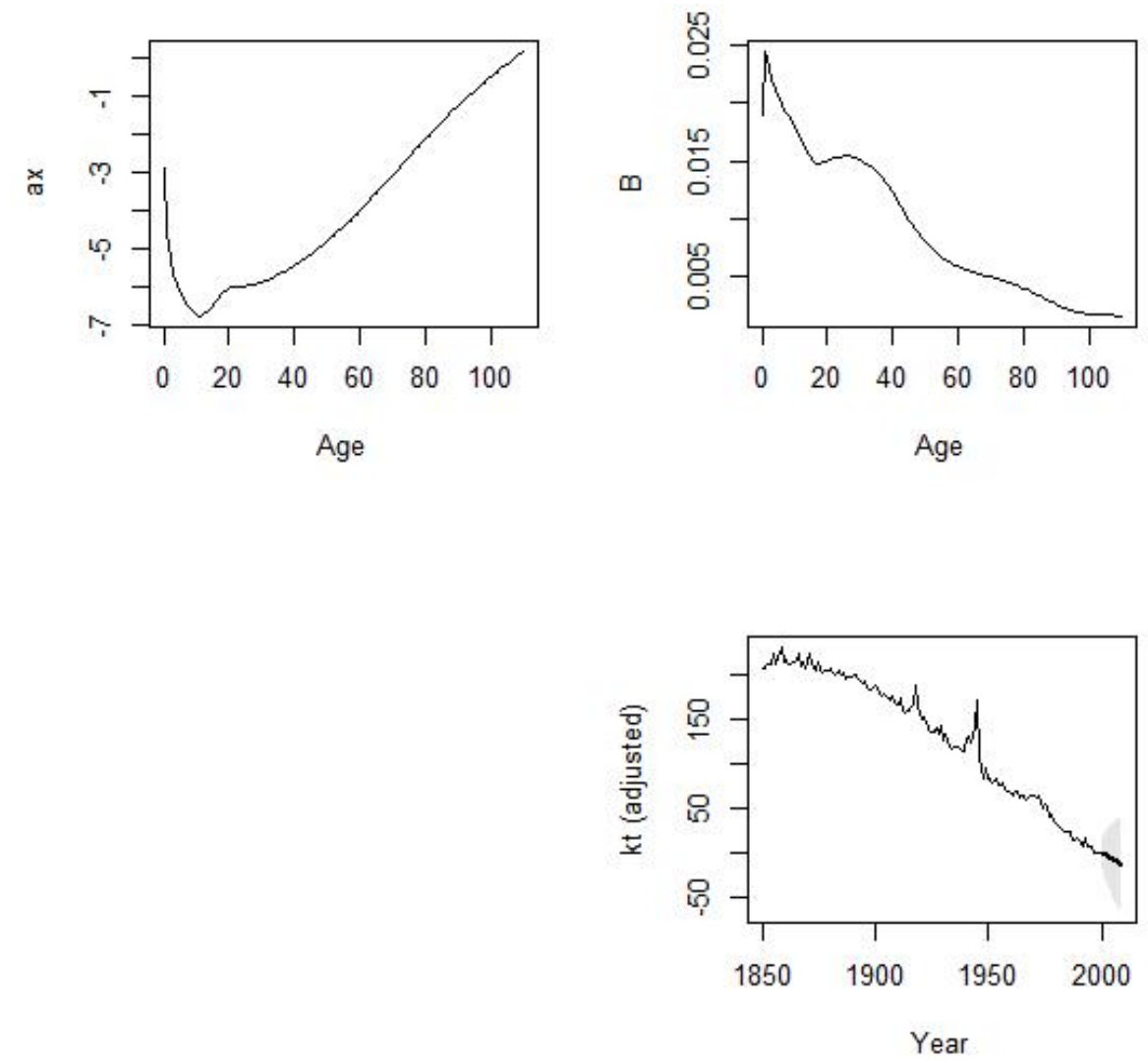

Figure 18 - Estimates for $a_{x}, b_{x}$ and the adjusted $k_{t}$ for the Netherlands, total population, "training set" 1850-1999. 
Mortality Modelling and Forecasting using Cross-Validation Techniques

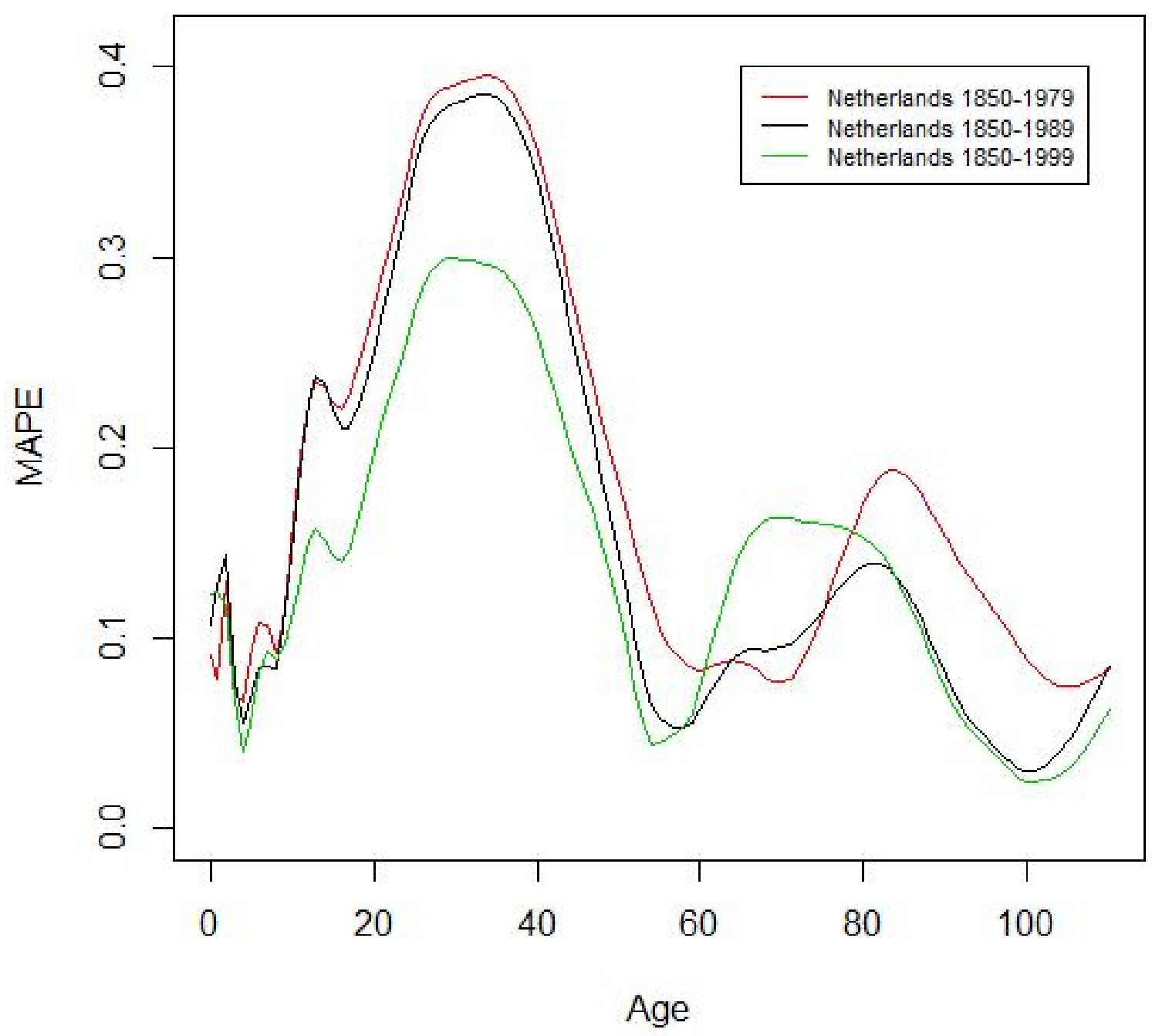

Figure 19 - Comparison MAPE Lee-Carter for the Netherlands, three different "training sets". 
Mortality Modelling and Forecasting using Cross-Validation Techniques
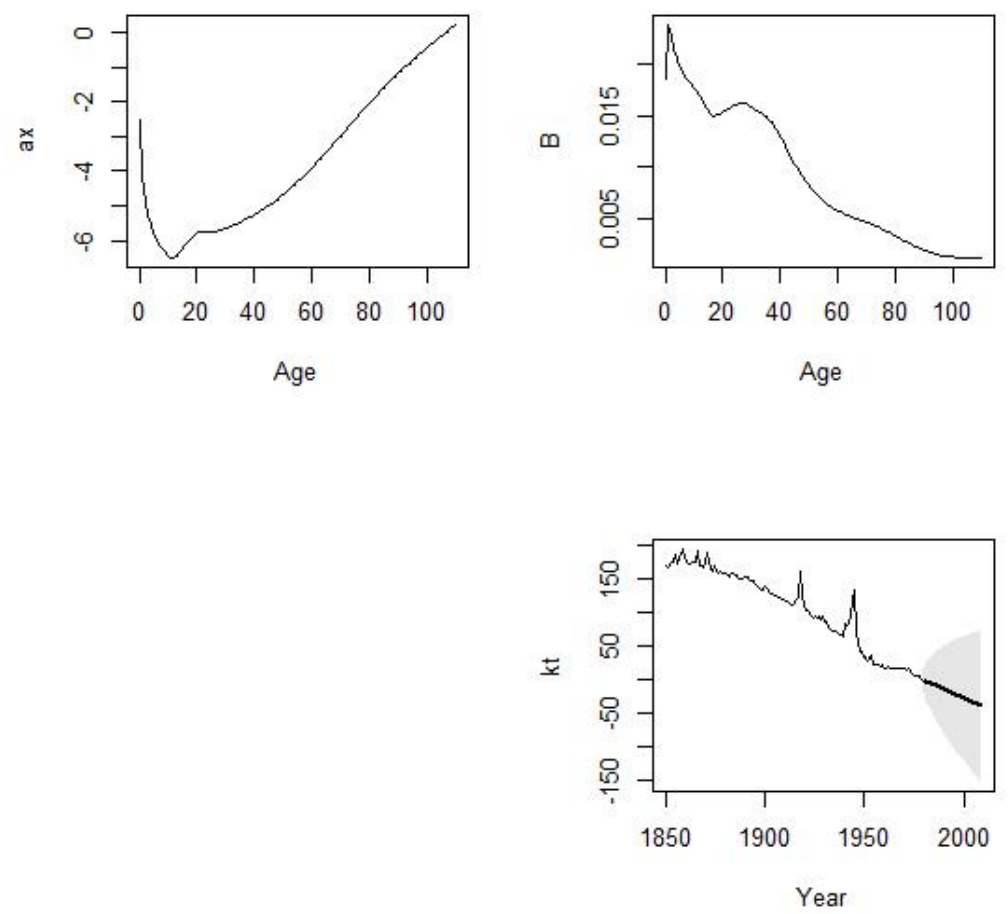

Figure 20 - Estimates for $a_{x}, b_{x}$ and the unadjusted $k_{t}$ for the Netherlands, total population. 
Mortality Modelling and Forecasting using Cross-Validation Techniques

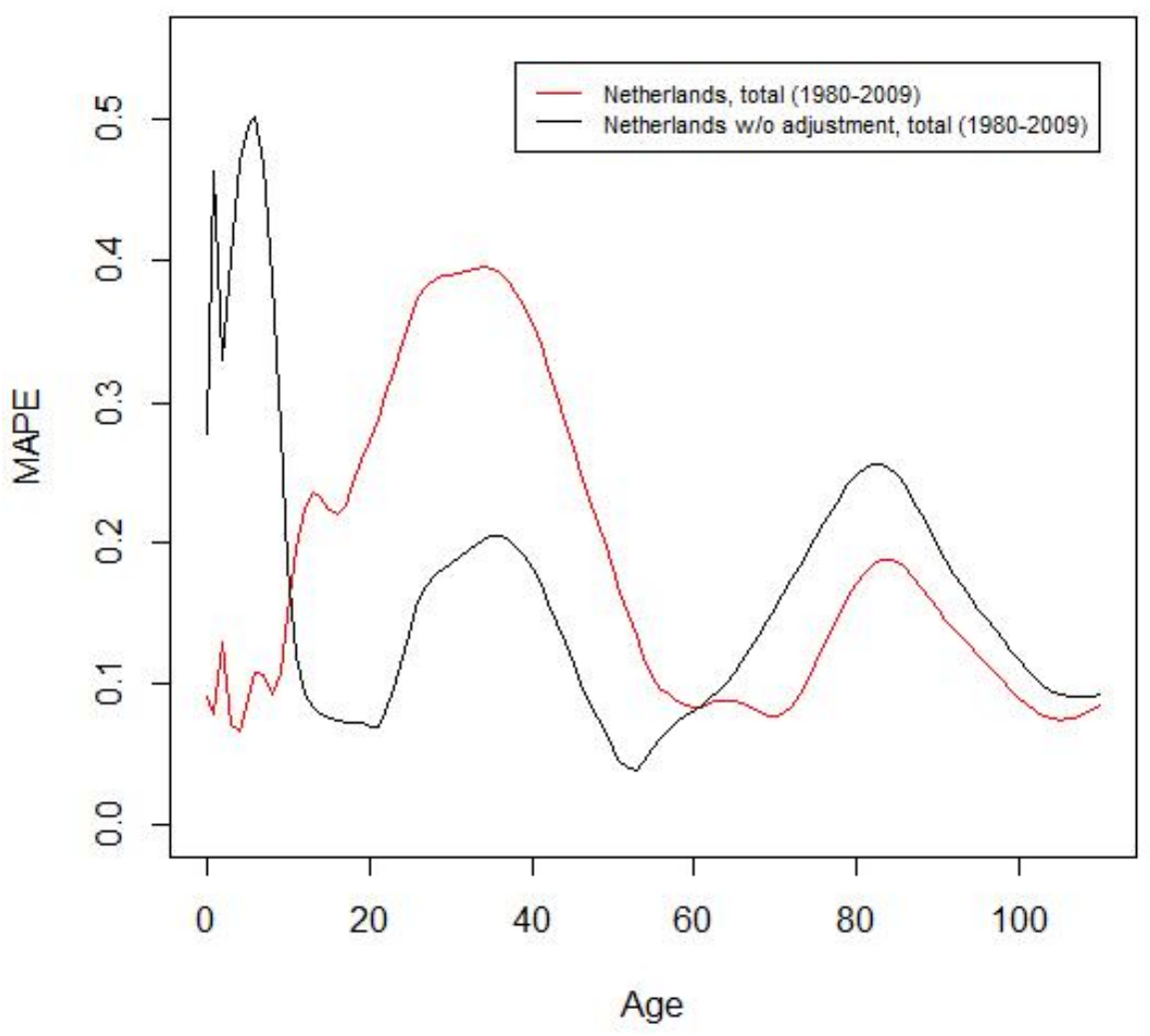

Figure 21 - Comparison MAPE Lee-Carter for forecasting without re-estimating $k_{t}$. 


\section{Mortality Modelling and Forecasting using Cross-Validation Techniques}

\section{Conclusions}

In this paper, the Heligman-Pollard model and the Lee-Carter model have been applied to the field of mortallity modelling and forecasting. Cross-validation techniques were used to measure how accurately these two models performed in practice. The main analysis in Chapter 3 was based on data from the Netherlands (total population) where the data set was divided into the "training set" (1850-1979) and the "testing set" (1980-2009).

The results, based on the MAPE, showed that the Heligman-Pollard model seemed to fit better to the Dutch data than the Lee-Carter model, although big errors at age 0 and $110_{+}$ occured. This is not in line with the general conclusion that parameterisation functions, such as the Heligman-Pollard model, usually do not perform well in forecasting mortality rates.

Chapter 4 focussed on the extensions: a distinction between the male and female population, extension to Danish and Norwegian population and the effects of increasing the size of the "training set" for the Lee-Carter model; and a weighted estimation for the Heligman-Pollard model. All these results were compared with the original results for both models with Dutch data.

The goal of the weighted Heligman-Pollard estimation was to reduce the big errors at age 0 and $110+$. The results showed that the error at age 0 was indeed significantly reduced, but on the other hand the error at age 110+ even slightly increased. Furthermore, big errors at age 14 and 15 arised.

The distinction between the Dutch male and female population for the Lee-Carter model led to remarkable results: high positive errors for the male population ages $0-20$ and high negative errors for the female population ages $0-60$, completely different from the MAPE for the total Dutch population.

Extending the Lee-Carter model to Denmark and Norway led to the conclusion that the Lee-Carter model seems to fit best to the Danish data: the big errors at adolescent ages were reduced significantly, but at ages 70-90 the MAPE increased. The Norwegian data fitted slightly better than the Dutch data, but large errors remained.

Investigating the effect of an increase in the "training set" was done by considering three cases: the original one, a 10-year increase and a 20 -year increase, leading to a forecast period of respectively 30,20 and 10 years. The results showed that increasing the "training set" leads to smaller errors at adolescent ages, but there was a significant increase in the MAPE at ages 60-80.

The last extension focussed on the effect of not using the adjustment for the $k_{t}$ series. This meant that the observed number of deaths each year would not be equal to the number of deaths observed by the model. The results showed that for ages 0-10 and 60-110+ there was an increase in the MAPE, whereas for adolescent ages the MAPE decreased. 


\section{Mortality Modelling and Forecasting using Cross-Validation Techniques}

\section{References}

[1] H. Booth, R.J. Hyndman, L. Tickle, and P. de Jong. Lee-carter mortality forecasting: a multicountry comparison of variants and extensions. Demographic Research, 15(9):289-310, 2006.

[2] $\mathrm{H}$. Booth and L. Tickle. Mortality modelling and forecasting: a review of methods. Annals of Actuarial Science, 3:3-43, 2008.

[3] L. Heligman and J.H. Pollard. The age pattern of mortality. Journal of the Institute of Actuaries, 107(434):49-80, 1980.

[4] R.J. Hyndman. demography: Forecasting mortality and fertility data, $r$ package. http: //www.robhyndman.info/Rlibrary/demography, 2006.

[5] R.D. Lee and L.R. Carter. Modelling and forecasting u.s. mortality. Journal of the American Statistical Association, 87(419):659-671, 1992.

[6] P. Refaeilzadeh, L. Tang, and H. Liu. Cross validation. Encyclopedia of Database Systems., Springer, 2009.

[7] J.R. Wilmoth. Computational methods for fitting and extrapolating the Lee-Carter model of mortality change. Technical report, Department of Demography, University of California, Berkeley, 1993. 